\title{
Uma medida de apego: versão brasileira da Experiences in Close Relationship Scale - Reduzida (ECR-R-Brasil)
}

\author{
Jean Carlos Natividade ${ }^{a^{*}}$ \\ Victor Kenji Medeiros Shiramizu ${ }^{\text {b }}$ \\ aPontifícia Universidade Católica do Rio de Janeiro, Programa de Pós-Graduação em Psicologia Clínica. Rio de Janeiro, RJ, Brasil \\ 'Universidade Federal do Rio Grande do Norte, Programa de Pós-Graduação em Psicobiologia. Natal, RN, Brasil
}

\begin{abstract}
Resumo: Os objetivos desta pesquisa foram adaptar e buscar evidências de validade para a população brasileira da versão reduzida da Experience in Close Relationship. Após procedimentos de tradução dos 12 itens, o instrumento foi respondido por 4.879 adultos de todas as regiões do país, $66 \%$ mulheres. Análises fatoriais exploratórias e confirmatórias evidenciaram a adequação da estrutura de dois fatores, em consonância com o estudo original. Os índices de consistência interna dos fatores "ansiedade" e "evitação" mostraram-se satisfatórios. Encontraramse resultados de diferenças sexuais e correlações com tempo de relacionamento e níveis de sociossexualidade de acordo com as hipóteses teóricas e muito semelhantes aos encontrados para a versão brasileira da medida estendida. Ainda, a escala foi capaz de diferenciar participantes que estavam em relacionamento compromissado daqueles em relacionamento sem compromisso. Considera-se que as evidências de validade e fidedignidade encontradas para a ECR-R-Brasil mostraram-se satisfatórias.
\end{abstract}

Palavras-chave: apego, comportamento de apego, amor, testes psicológicos, validade do teste.

\section{Introdução}

O conceito de apego diz respeito ao vínculo afetivo/emocional estabelecido entre seres humanos, em que um deles representa uma figura de apego e o outro busca proximidade a essa figura em situações de desconforto ou ameaça (Ainsworth, 1989). Bowlby (1969/2002; 1973/2004; 1980/1998), um dos pioneiros na conceituação e estudo do apego, elaborou sua teoria a partir da investigação dos vínculos estabelecidos entre crianças e seus cuidadores. Seus postulados sobre a importância dos vínculos iniciais entre cuidador e criança inspiraram estudos que demonstraram como a qualidade da relação com o cuidador principal poderia culminar em singularidades comportamentais nas crianças, o que poderia ser expresso por diferentes estilos de apego (Ainsworth, 1989). Posteriormente, iniciaram-se investigações sobre o impacto dos estilos de apego estabelecidos na infância e suas repercussões nas interações na vida adulta (Hazan \& Shaver, 1987; Shaver, Hazan, \& Bradshaw, 1988).

Hazan e Shaver (1987) transpuseram a noção do vínculo estabelecido na infância para os estabelecidos em relacionamentos amorosos. Os autores desenvolveram uma medida de autorrelato destinada a aferir três estilos de apego em adultos. A partir da medida, verificaram que indivíduos com um estilo de apego seguro percebiam a relação com o parceiro como mais afetuosa, amigável e confiante. Já indivíduos com estilos de apego inseguro ansioso (que remete a um cuidado inconsistente durante a infância) relataram uma

* Autor correspondente: jeannatividade@gmail.com maior necessidade de desejo por união e reciprocidade com o parceiro romântico. Por último, indivíduos apresentando um estilo de apego inseguro evitativo (associado com um cuidado insensível durante a infância) relataram sentir-se incomodados com a proximidade física e emocional com o parceiro romântico.

O trabalho de Hazan e Shaver (1987) abriu as portas de um novo campo para a investigação do apego; contudo, o instrumento de medida utilizado pelos autores recebeu muitas críticas. Entre as principais críticas destaca-se aquela sobre o caráter de escolha forçada do instrumento, classificando os indivíduos de forma categórica, não sendo possível estabelecer gradações entre os estilos de apego (Feeney, 2008). Outra crítica semelhante ao instrumento refere-se à impossibilidade de obtenção de informações precisas para avaliar diferenças individuais (Fraley \& Shaver, 2000). Muito em decorrência das críticas, diversos instrumentos para aferir apego adulto têm sido desenvolvidos como uma alternativa para aferir o apego de maneira contínua e dimensional (Fraley \& Shaver, 2000).

Atualmente, uma medida frequentemente utilizada na pesquisa sobre apego adulto no contexto dos relacionamentos amorosos é a escala denominada Experience in Close Relationships (ECR) de Brennan, Clark e Shaver (1998). Para citar alguns exemplos de sua replicabilidade, a ECR já foi traduzida e adaptada para populações italiana (Piccardi, Bitetti, Puddu, \& Pasquini, 2000), chinesa (Mallinckrodt \& Wang, 2004), espanhola (Alonso-Arbiol, Balluerka, \& Shaver, 2007), portuguesa (Paiva \& Figueiredo, 2010) e brasileira (Shiramizu, Natividade, \& Lopes, 2013). Em geral, as adaptações têm mostrado satisfatórios índices de ajuste à 
estrutura original e evidências de validade de natureza convergente e discriminante, além de adequados coeficientes de consistência interna.

A ECR é uma escala de autorrelato composta por 36 itens provenientes de análises fatoriais realizadas com itens de vários instrumentos sobre apego disponíveis à época (Brennan et al., 1998). Os autores encontraram duas dimensões ortogonais explicativas do apego, tais como: "ansiedade relacionada ao apego" e "evitação relacionada ao apego". A primeira dimensão diz respeito à preocupação com a continuidade do relacionamento amoroso e com a responsividade do parceiro, bem como, a uma necessidade de proximidade física e emocional. Já a segunda dimensão refere-se ao desconforto com a proximidade emocional, com a dependência de parceiros românticos, uma preferência por distanciamento emocional.

Ainda que a ECR possa ser considerada consistente e adequada, a preocupação com sua extensão (36 itens) e a necessidade de uma medida que pudesse ser respondida rapidamente em diversos contextos levou Wei, Russell, Mallinckrodt e Vogel (2007) a desenvolverem uma escala reduzida para apego adulto. Os autores denominaram a escala de ECR-Short (ECR-S) em função de sua construção a partir dos itens da ECR de Brennan et al. (1998). A ECR-S é uma escala de 12 itens que afere as duas dimensões do apego. A escala reduzida apresentou adequados índices de ajuste à estrutura de dois fatores e consistência interna satisfatória para as duas dimensões.

A importância de uma medida mais curta e rápida de ser respondida recai, principalmente, na facilidade de aplicação de um instrumento com essas características em diversos contextos de pesquisa. Uma medida reduzida pode contribuir, por exemplo, para a diminuição da taxa de desistência de participantes em pesquisas que já contêm um razoável número de questões a serem respondidas. Considerando a carência de instrumentos curtos com adequadas evidências de validade para a população brasileira para aferir apego adulto, delineou-se esta pesquisa com os objetivos de adaptar e buscar evidências de validade para a população brasileira da versão reduzida da ECR, nomeadamente a ECR-S de Wei et al. (2007).

\section{Método}

\section{Participantes}

Participaram 4.879 pessoas provenientes das cinco regiões do Brasil, com média de idade de 27,8 anos ( $D P=8,98)$, $66 \%$ eram mulheres. Quanto à escolaridade, a maioria dos participantes tinha até ensino superior incompleto $(52,4 \%)$; os demais tinham ensino superior completo, sendo que $12,1 \%$ tinham pós-graduação incompleta, e $20,4 \%$, pós-graduação completa. A maior parte dos participantes estava na região Sul do país (52,7\%); a região Sudeste contou com 23,9\% dos respondentes; a região Nordeste, com 12,9\%; a Centro-Oeste com 5,5\%; do Norte foram 3,6\%; os demais $(1,4 \%)$ estavam fora do Brasil.

\section{Instrumentos}

Utilizou-se um questionário disponibilizado em um endereço na internet com questões sociodemográficas (sexo, idade, escolaridade, local de residência) e questões sobre relacionamentos amorosos, tais como: número de relacionamentos ao longo da vida, tempo de duração do relacionamento mais longo que já vivenciou, se estava em um relacionamento atualmente e qual o tempo de duração do relacionamento atual. Além dessas perguntas, o questionário continha os seguintes instrumentos:

\section{Escala Experience in Close Relationship - Reduzida (ECR-R-Brasil)}

Esse instrumento foi traduzido e adaptado para o Brasil neste estudo a partir do instrumento de Wei et al. (2007). O original contém 12 itens, seis referentes à dimensão "ansiedade relacionada ao apego" e seis referentes à dimensão "evitação relacionada ao apego". Os itens são frases afirmativas para que os participantes respondam o quanto concordam em uma escala de sete pontos. A versão final do instrumento adaptado para o Brasil conta com 10 itens, cinco para cada dimensão do apego, maiores escores indicam maiores níveis de "ansiedade" e "evitação" relacionada ao apego.

Inventário de Orientação Sociossexual - Revisado (Natividade, Fernandes, \& Hutz, 2013)

Esse instrumento afere níveis de restrição/irrestrição sexual a partir de três dimensões: comportamento, atitude e desejo. Trata-se de uma versão traduzida e adaptada para o Brasil do Revised Sociosexual Orientation Inventory (Penke \& Asendorpf, 2008). O instrumento conta com nove itens para serem respondidos em uma escala de nove pontos, tal que quanto maiores os escores, maior a irrestrição sexual. A adaptação do instrumento à realidade brasileira contou com participantes das cinco regiões do país $(N=1887)$, apresentou satisfatórias evidências de validade e coeficientes alfa variando de 0,85 a 0,89 .

Escala de Desejabilidade Social (Ribas, Seidl de Moura, \& Hutz, 2004)

A Escala de Desejabilidade Social utilizada corresponde à versão brasileira da escala abreviada de Crowne e Marlowe (1960), que afere uma tendência a responder os itens de forma que possa ser considerada desejável perante a sociedade. Essa escala é formada por 13 afirmativas para que os participantes respondam se consideram se os itens os descrevem verdadeiramente ou não. No estudo de adaptação para o Brasil, o instrumento apresentou coeficiente alfa de 0,70 (Ribas et al., 2004). Escores altos na escala indicam alta desejabilidade social.

Junto dos itens de cada escala, incluíram-se questões de controle em que se solicitava ao participante responder de 
maneira específica, por exemplo: "essa é uma questão controle, por favor, assinale o número cinco na escala". Essas questões foram usadas para excluir possíveis respostas aleatórias. O questionário foi configurado para não permitir respostas omissas aos itens das escalas.

\section{Procedimentos}

\section{Tradução da Escala Experience in Close Relationship - Reduzida (ECR-R-Brasil)}

Inicialmente, duas pessoas bilíngues traduziram, independentemente, o instrumento de Wei et al. (2007) do inglês para o português. Em um segundo momento, uma terceira pessoa bilíngue e com experiência em adaptação de instrumentos psicológicos comparou as versões traduzidas com o original em inglês, e então se compilou uma versão unificada dos itens em português. Buscaram-se traduções para os itens que resguardassem ao máximo a representatividade do conteúdo em inglês, levando-se em conta a teoria sobre apego e definições das dimensões avaliadas. Então, apresentou-se a versão traduzida a um grupo de 24 pessoas para que julgassem a adequação da redação dos itens. A partir disso, fizeram-se ajustes de redação e elaborou-se a versão do instrumento para ser posta à prova empírica.

\section{Coleta de dados}

O recrutamento dos participantes ocorreu de maneira mista: presencial e via internet. $\mathrm{O}$ recrutamento presencial deu-se conforme procedimento de coleta on-line com recrutamento presencial (Wachelke, Natividade, De Andrade, Wolter, \& Camargo, 2014). A partir desse procedimento coletaram-se endereços de e-mails de estudantes de diversos cursos de graduação em universidades públicas do Sul e Sudeste do Brasil. Posteriormente, encaminharam-se e-mails de convite aos estudantes contendo o link do questionário. $\mathrm{O}$ recrutamento via internet deu-se por meio de e-mails de convite e divulgação em redes sociais. A pesquisa foi desenvolvida de acordo com as recomendações éticas de pesquisa com seres humanos e obteve-se a aprovação do Comitê de Ética em Pesquisa do Instituto de Psicologia da Universidade Federal do Rio Grande do Sul, sendo parte do projeto de pesquisa protocolado sob número 22035.

\section{Resultados}

Inicialmente foi realizada a limpeza dos dados, excluindo-se da amostra as respostas erradas às questões controle e outras respostas claramente falseadas, por exemplo a mesma resposta para todos os itens de todas as escalas do questionário. Em seguida, conduziu-se uma análise de componentes principais com os itens da ECR-R-Brasil. Constatou-se adequação dos dados à análise, $K M O=0,80$; teste de esfericidade de Bartlett, $\chi^{2}(66, N=4879)=13.309$; $p<0,001$. A Tabela 1 mostra as cargas componenciais dos itens para as duas dimensões do apego. Observa-se que os itens agruparam-se de acordo com a proposta inicial de Wei et al. (2007), os itens 2, 4, 6, 8, 10 e 12 apresentaram cargas mais altas no primeiro componente, representativo da

Tabela 1

Cargas componenciais dos itens da ECR-R - Brasil a partir de uma análise de componentes principais

\begin{tabular}{|c|c|c|c|}
\hline Itens & Ansiedade & Evitação & $h^{2}$ \\
\hline 2. Eu preciso de muitas garantias de que sou amado por meu(minha) parceiro(a). & 0,71 & $-0,13$ & 0,53 \\
\hline $\begin{array}{l}\text { 12. Preocupa-me que meu(minha) parceiro(a) não se importe comigo tanto quanto eu me } \\
\text { importo com ele(a). }\end{array}$ & 0,71 & 0,01 & 0,51 \\
\hline $\begin{array}{l}\text { 4. Frequentemente, eu acho que meu(minha) parceiro(a) não quer tanta proximidade } \\
\text { afetiva quanto eu gostaria. }\end{array}$ & 0,66 & 0,29 & 0,52 \\
\hline 6. Às vezes, meu desejo de ficar muito próximo afetivamente acaba assustando as pessoas. & 0,64 & 0,10 & 0,42 \\
\hline 10. Eu fico frustrado se meu(minha) parceiro(a) não está disponível quando eu preciso dele. & 0,64 & $-0,17$ & 0,43 \\
\hline 8. Geralmente, não me preocupo em ser abandonado por um(a) parceiro(a). & $-0,42$ & 0,23 & 0,23 \\
\hline 9. Eu costumo conversar sobre os meus problemas e preocupações com meu(minha) parceiro(a). & 0,05 & $-0,70$ & 0,49 \\
\hline 7. Geralmente, tento evitar muita proximidade afetiva com meu(minha) parceiro(a) & 0,11 & $\mathbf{0 , 7 0}$ & 0,50 \\
\hline 1. Ajuda muito poder contar com meu(minha) parceiro(a) em momentos de necessidade. & 0,20 & $-0,67$ & 0,50 \\
\hline $\begin{array}{l}\text { 5. Eu recorro ao(à) meu(minha) parceiro(a) para muitas coisas, incluindo para conforto e } \\
\text { segurança emocional. }\end{array}$ & 0,27 & $-0,67$ & 0,53 \\
\hline 11. Eu fico preocupado quando meu(minha) parceiro(a) fica muito próximo afetivamente de mim. & 0,10 & 0,65 & 0,43 \\
\hline $\begin{array}{l}\text { 3. Geralmente, eu quero me aproximar afetivamente de meu(minha) parceiro(a), mas eu } \\
\text { acabo me distanciando. }\end{array}$ & 0,46 & 0,53 & 0,49 \\
\hline $\begin{array}{ll}\text { Eigenvalues }\end{array}$ & 2,80 & 2,77 & \\
\hline \% Variância explicada & 23,3 & 23,1 & \\
\hline
\end{tabular}

Nota: N=4879. Método de rotação varimax. A numeração dos itens está em acordo com a versão de Wei et al. (2007); a versão final do instrumento para o Brasil apresenta numeração diferente, tendo-se em vista a exclusão dos itens 3 e 8 acima. Cargas maiores que 0,30 estão em negrito 
"ansiedade", enquanto os itens 1, 3, 5, 7, 9 e 11 mostraram cargas mais elevadas no segundo componente, representativo da "evitação". A correlação entre os dois componentes foi de $-0,04$. Essa análise inicial permitiu a sinalização de possíveis itens problemáticos, como o item 3, que apresentou carga semelhante nos dois componentes, e 0 item 8 , com baixo índice de comunalidade.

O passo seguinte foi testar a adequação estrutural do instrumento de modo confirmatório, bem como comparar modelos alternativos semelhante ao de Wei et al. (2007) e excluir os itens problemáticos evidenciados na análise exploratória. Para a estimação dos parâmetros partiu-se da matriz de covariância dos dados e optou-se pelo algoritmo Maximum Likelihood. Utilizou-se o software AMOS 16 nessas análises e avaliaram-se os índices de ajuste que têm sido apontados como relevantes pela literatura especializada (Byrne, 2009; Jackson, Gillaspy Jr., \& Purc-Stephenson, 2009). A Tabela 2 mostra os índices de ajuste encontrados.

Observam-se índices de ajustes mais adequados para os modelos configurados com a inclusão de dois fatores artificiais, tal como propuseram Wei et al. (2007) no estudo original da versão reduzida da ECR. Ainda, pode-se constatar um melhor ajuste para o modelo sem os itens 3 e 8 da Tabela 1. Uma inspeção nos índices de correlação item-total também permite constatar um leve incremento no coeficiente alfa com a retirada do item 8 da "ansiedade", de 0,72 para
0,73 ; e nenhuma alteração com a retirada do item 3 da "evitação", permanecendo um coeficiente de 0,73 . Tais resultados apontam para a satisfatória adequação do instrumento de 10 itens para o Brasil, cinco para cada dimensão. As análises seguintes foram conduzidas utilizando-se essa configuração de 10 itens.

O passo seguinte em busca de evidências de validade para o instrumento foi testar correlações entre as dimensões de apego aferidas pela ECR-R-Brasil, sociossexualidade e questões critério sobre relacionamentos amorosos, tal como fizeram Shiramizu et al. (2013) para a ECR-Brasil estendida. A Tabela 3 mostra os coeficientes de correlação de Pearson encontrados para homens e mulheres. Destacam-se coeficientes muito semelhantes aos encontrados para a versão estendida da ECR para o Brasil.

Adicionalmente, testou-se a diferença dos coeficientes de correlação observados neste estudo com a medida reduzida (ECR-R-Brasil), e aqueles relatados por Shiramizu et al. (2013) para a medida estendida no Brasil, utilizando-se teste Z de Fisher (1921). Compararam-se os coeficientes das duas medidas para as correlações entre apego e três dimensões de sociossexualidade, o tempo de relacionamento mais longo e o tempo de relacionamento atual. Verificaramse diferenças significativas entre as duas medidas para dois coeficientes: entre os homens, o coeficiente de correlação da "ansiedade" com a dimensão "desejo da sociossexualidade"

Tabela 2

Índices dos modelos testados a partir de análise fatorial confirmatória

\begin{tabular}{ccccc}
\hline \multicolumn{3}{c}{ Modelos } \\
\hline & $\begin{array}{c}\text { Inicial } \\
(12 \text { itens })\end{array}$ & Modificado (10 itens) & Inicial-artificios & Modificado-artifícios \\
\hline$\chi^{2}$ & 3545,0 & 2118,9 & 1145,2 & 101,8 \\
gl & 53 & 34 & 41 & 25 \\
$\mathrm{p}$ & $<0,001$ & $<0,001$ & $<0,001$ & $<0,001$ \\
X2/g1 & 66,9 & 62,3 & 27,9 & 4,07 \\
GFI & 0,88 & 0,92 & 0,96 & 0,99 \\
AGFI & 0,82 & 0,86 & 0,93 & 0,99 \\
NFI & 0,73 & 0,80 & 0,91 & 0,99 \\
TLI & 0,67 & 0,75 & 0,87 & 0,99 \\
CFI & 0,74 & 0,81 & 0,92 & 0,99 \\
RMESA & 0,116 & 0,112 & 0,074 & 0,025 \\
IC 90\% RMESA & $0,113-0,119$ & $0,108-0,116$ & $0,071-0,078$ & $0,020-0,030$ \\
CAIC & 3782,4 & 2318,3 & 1515,4 & 415,0 \\
\hline
\end{tabular}

Nota: Inicial - Modelo configurado com dois fatores correlacionados, um composto por seis itens referentes à "ansiedade" (os itens com numeração par da Tabela 1 deste estudo) e outro com seis itens referentes à "evitação" (os itens com numeração ímpar da Tabela 1), de acordo com Wei et al. (2007). Modificado - Modelo configurado do mesmo modo que o modelo Inicial, porém sem os itens 3 e 8 da Tabela 1. Inicial-artifícios - Modelo configurado como o inicial e acrescentado dois fatores, um composto pelos itens reversos (itens 1, 5, 8 e 9) e outro pelos não-reversos (itens 2, 3, 4, 6, 7, 10, 11, 12 da Tabela 1), idêntico ao de Wei et al. (2007). Modificado-artifícios - Modelo configurado de modo semelhante ao Inicial-artifícios, porém sem os itens 3 e 8 da Tabela 1. $\chi^{2}$ - qui-quadrado; $g l$ - graus de liberdade; $\chi^{2} / g l$ - razão qui-quadrado por graus de liberdade; GFI - Goodness-of-Fit Index; AGFI - Adjusted Goodness-of-Fit Index; NFI - Normed Fit Index; TLI - Tucker-Lewis Index; CFI - Comparative Fit Index; RMSEA - Root Mean Square Error of Aproximation; IC 90\% RMESA - Intervalo de confiança de 90\%; CAIC - Consistent Akaike Information Criterion 
Tabela 3

Relações entre fatores de apego ansiedade e evitação e demais variáveis investigadas para homens e mulheres

\begin{tabular}{|c|c|c|c|c|c|c|c|c|c|c|}
\hline & 1 & 2 & $\begin{array}{c}3 \\
n=1113\end{array}$ & $\begin{array}{c}4 \\
n=1509\end{array}$ & $\begin{array}{c}5 \\
n=1534\end{array}$ & 6 & 7 & 8 & 9 & 10 \\
\hline $\begin{array}{l}\text { 1. ECR-R Brasil - } \\
\text { "ansiedade" (cinco itens) }\end{array}$ & - & $-0,07 *$ & $-0,16^{*}$ & $-0,19 *$ & 0,02 & $-0,04$ & $-0,13^{*}$ & $0,09 *$ & $-0,22 *$ & $-0,12 *$ \\
\hline $\begin{array}{l}\text { 2. ECR-R Brasil - } \\
\text { "evitação" (cinco itens) }\end{array}$ & 0,02 & - & $-0,18^{*}$ & $-0,21 *$ & 0,06 & $0,11^{*}$ & $0,17^{*}$ & $0,21^{*}$ & $-0,02$ & $-0,06^{*}$ \\
\hline $\begin{array}{l}\text { 3. Tempo relacionamento } \\
\text { atual } \dagger, n=2313\end{array}$ & $-0,13^{*}$ & $-0,25^{*}$ & - & $0,71^{*}$ & $-0,06^{*}$ & $-0,12 *$ & 0,00 & $-0,08^{*}$ & $0,09 *$ & $0,48^{*}$ \\
\hline $\begin{array}{l}\text { 4. Tempo relacionamento } \\
\text { mais longo } \dagger, n=3059\end{array}$ & $-0,11^{*}$ & $-0,26^{*}$ & $0,65^{*}$ & - & $0,15^{*}$ & $0,15^{*}$ & $0,05^{*}$ & $-0,08^{*}$ & $0,07 *$ & $0,61 *$ \\
\hline $\begin{array}{l}\text { 5. Número de } \\
\text { relacionamentos na vida } \\
\dagger, n=3096\end{array}$ & $0,07 *$ & $0,08^{*}$ & $-0,12 *$ & $0,11^{*}$ & -- & $0,28^{*}$ & $0,12^{*}$ & $0,09^{*}$ & 0,01 & $0,22 *$ \\
\hline $\begin{array}{l}\text { 6. SOI-R Brasil - } \\
\text { Comportamento }\end{array}$ & $0,11^{*}$ & $0,14^{*}$ & $-0,24 *$ & 0,04 & $0,35^{*}$ & - & $0,44^{*}$ & $0,30^{*}$ & $-0,05$ & $0,28^{*}$ \\
\hline 7. SOI-R Brasil - Atitude & $-0,004$ & $0,12 *$ & $-0,13 *$ & 0,01 & $0,19^{*}$ & $0,57^{*}$ & -- & $0,48^{*}$ & $-0,14^{*}$ & 0,03 \\
\hline 8. SOI-R Brasil - Desejo & $0,12^{*}$ & $0,26^{*}$ & $-0,24 *$ & $-0,14 *$ & $0,16^{*}$ & $0,38 *$ & $0,50^{*}$ & -- & $-0,16^{*}$ & $-0,08^{*}$ \\
\hline $\begin{array}{l}\text { 9. Desejabilidade social, } \\
\mathrm{n}=4671\end{array}$ & $-0,24 *$ & $-0,03$ & $0,08^{*}$ & $0,07 *$ & $-0,06^{*}$ & $-0,13 *$ & $-0,21 *$ & $-0,19^{*}$ & -- & $0,08^{*}$ \\
\hline 10. Idade & $-0,05^{*}$ & $0,05^{*}$ & $0,47^{*}$ & $0,59^{*}$ & $0,24^{*}$ & $0,13^{*}$ & 0,01 & $-0,06^{*}$ & $0,08^{*}$ & -- \\
\hline
\end{tabular}

Nota: $* \mathrm{p}<0,01$. Acima da diagonal principal apresentam-se os coeficientes de correlação para os homens, abaixo para as mulheres. Quando não indicado, $\mathrm{N}=3221$ mulheres e N=1658 homens. † Controlou-se o efeito da idade nas correlações com Apego para essas variáveis. Utilizaram-se transformações logarítmicas nos cálculos dos coeficientes de correlação das variáveis 'Tempo de relacionamento atual' e ‘Tempo de relacionamento mais longo', tendo-se em vista a alta dispersão dos dados. Coeficientes de correlação maiores, ou igual, a 0,30 estão em negrito

foi de $r(1698)=0,09$ para a medida reduzida e $r(252)=-0,09$ para a medida estendida, teste $\mathrm{Z}$ de Fisher, $z=2,63 ; p=0,008$; entre as mulheres, o coeficiente de correlação da "evitação" com o "tempo de relacionamento mais longo" para a medida reduzida foi de $r(3221)=-0,15$, e para a medida estendida foi de $r(366)=-0,30$, teste $\mathrm{Z}$ de Fisher, $z=2,83 ; p=0,005$.

Por fim, testamos diferenças para as dimensões de apego entre homens e mulheres e entre pessoas que estavam em relacionamento compromissado e sem compromisso. As diferenças sexuais para apego foram significativas, $\Lambda$ de Wilks $=0,95 ; F(2,4876)=133,7 ; p<0,001 ; \eta^{2}=0,052$. As análises univariadas conduzidas em seguida mostraram que as mulheres pontuaram mais que os homens em "ansiedade", $F(1,4877)=59,6 ; p<0,001 ; d=0,23$; e os homens tiveram maiores escores que as mulheres em "evitação", $F(1$, $4877)=210,8 ; p<0,001 ; d=0,43$. No que diz respeito às diferenças entre grupos de pessoas em relacionamento compromissado e sem compromisso, as diferenças também foram significativas, $\Lambda$ de Wilks $=0,89 ; F(2,3455)=222,9 ; p<0,001$; $\eta^{2}=0,11$. As análises univaridas evidenciaram maiores médias em "ansiedade", $F(1,3456)=69,8 ; p<0,001 ; d=0,40$, e "evitação", $F(1,3456)=358,4 ; p<0,001 ; d=0,87$, entre aqueles em relacionamento sem compromisso. As médias podem ser visualizadas na Figura 1.

A fim de fornecer escores normatizados para o instrumento elaborou-se a Tabela 4, em que se apresentam os percentis e as médias para homens e mulheres. Salienta-se que esses escores devem ser interpretados levando-se em conta que quase metade da amostra é de pessoas com nível superior completo.

\section{Discussão}

Acredita-se ter encontrado, neste estudo, satisfatórias evidências de validade da ECR-R-Brasil. Inicialmente, verificou-se a adequação da estrutura de dois fatores ortogonais para o instrumento, tanto a partir de análise exploratória, quanto confirmatória. O resultado está em acordo com a teoria que sustenta o instrumento e com a versão original da escala de Wei et al. (2007), bem como com a versão estendida do instrumento para o Brasil (Shiramizu et al., 2013). As dimensões aferidas dizem respeito à "ansiedade" (preocupação com a responsividade do parceiro e com a estabilidade do relacionamento em geral, bem como recorrente necessidade de proximidade física e emocional) e à "evitação" (desconforto com a proximidade emocional, com a dependência de parceiros amorosos e uma preferência por distanciamento emocional).

Destaca-se a exclusão de dois itens, um de cada dimensão do apego, para a versão brasileira do instrumento aqui adaptada. Ainda que os resultados de ajuste para o modelo idêntico ao de Wei et al. (2007), com 12 itens e dois 
fatores artificiais, pudessem ser considerados adequados, observou-se um melhor ajuste ao retirarem-se dois itens identificados como problemáticos na análise exploratória. Os índices de consistência interna dos fatores mostraram-se satisfatórios (Cronbach, 1951; Nunnally, 1978), e observou-se um leve incremento ao se retirar um dos itens identificados como problemáticos. Assim, a ECR-R-Brasil conta com 10 itens, cinco referentes à "ansiedade" e cinco referentes à "evitação" relacionada ao apego (Anexo 1).

$\mathrm{O}$ instrumento, em seus dois fatores, mostrou fracas correlações com desejabilidade social, o que fortalece suas evidências de validade e utilidade. Ao mesmo tempo que se constata que a escala é capaz de captar divergência de outro construto acessado, verifica-se que está pouco suscetível às pressões normativas para o endosso de itens. Também em busca de novas evidências de validade, testaram-se correlações de apego e sociossexualidade, número de relacionamentos amorosos ao longo da vida, tempo do relacionamento amoroso atual e tempo do relacionamento amoroso mais longo na vida. Contudo, todos os coeficientes de correlação encontrados podem ser considerados fracos no sentido esperado pela teoria e estudos anteriores (Allen \& Baucom, 2004; Del Guidice, 2009; Feeney \& Noller, 1990; Gentzler \& Kerns, 2004; Kirkpatrick \& Hazan, 1994; Jackson \& Kirkpatrick, 2007; Schmitt, 2005; Simpson, 1990; Shiramizu et al., 2013).

Por exemplo, neste estudo encontraram-se correlações positivas entre as três dimensões da sociossexualidade e a "evitação relacionada ao apego", tanto para homens quanto para mulheres. Tais resultados indicam que uma orientação sociossexual irrestrita, e consequentemente uma estratégia reprodutiva de curto prazo, está associada a um estilo de apego evitativo, o que vai ao encontro dos achados de Gentzler e Kerns (2004), Jackson e Kirkpatrick (2007), Schmitt (2005) e Shiramizu et al. (2013). Outros resultados que estão de acordo com essa noção, e em consonância com o que acharam Kirkpatrick e Hazan (1994), são aqueles relativos às

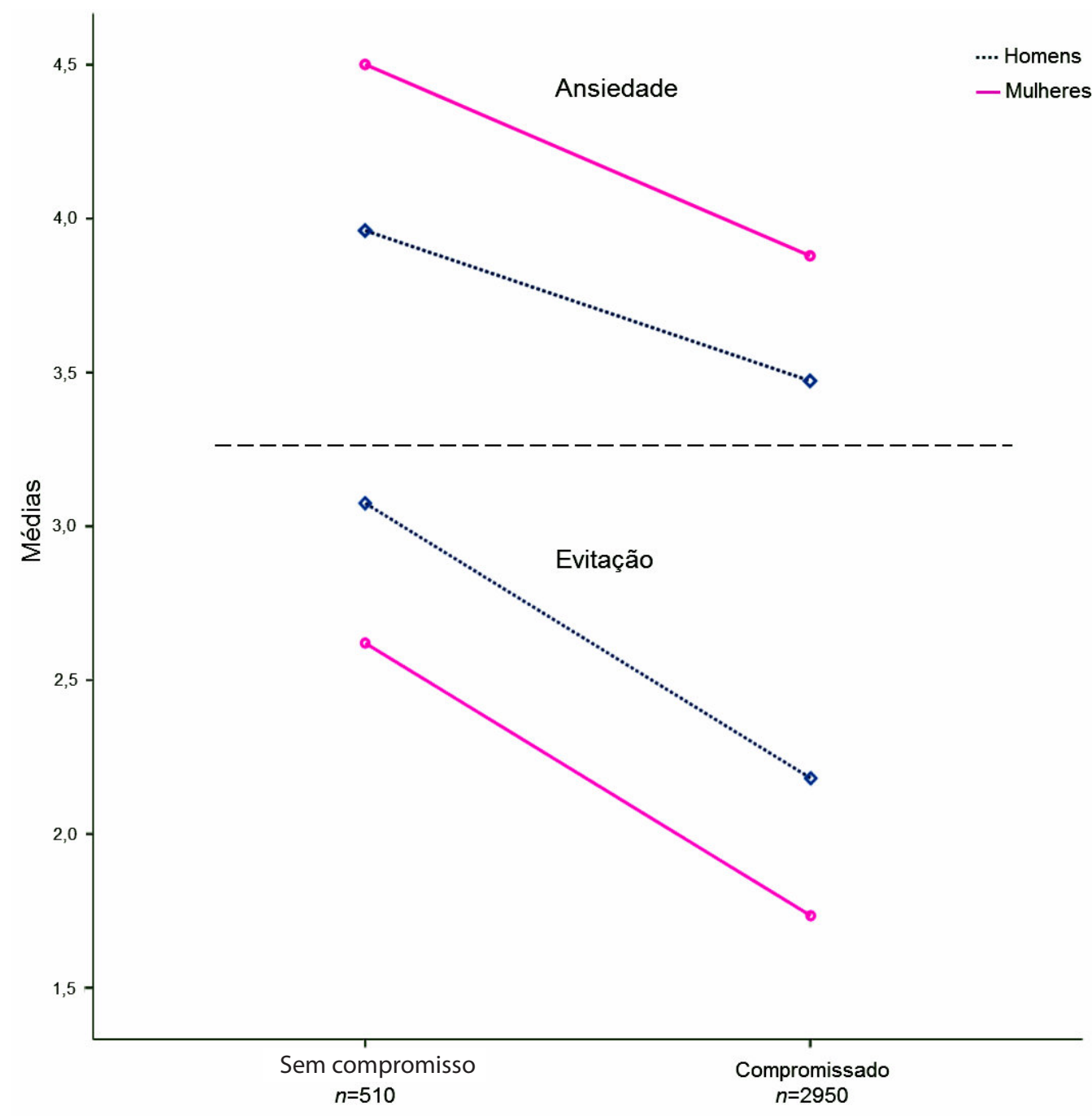

Figura 1. Médias nos fatores de "apego", "ansiedade" e "evitação" para homens e mulheres em relacionamento. $N=2950$, sendo 2334 mulheres e 1126 homens 
correlações negativas entre as duas dimensões do apego e tempo de relacionamento atual e tempo de relacionamento mais longo.
Além de se constatarem relações esperadas entre os fatores aferidos pela ECR-R-Brasil e variáveis e construtos correlatos, verificaram-se coeficientes de correlação

Tabela 4

Percentis, médias e desvios-padrão para "ansiedade" e "evitação" (ECR-R-Brasil) por sexo

\begin{tabular}{|c|c|c|c|c|c|c|}
\hline & \multicolumn{3}{|c|}{ Ansiedade } & \multicolumn{3}{|c|}{ Evitação } \\
\hline & Mulher & Homem & Todos & Mulher & Homem & Todos \\
\hline $\mathrm{N}$ & 3221 & 1658 & 4879 & 3221 & 1658 & 4879 \\
\hline M & 4,10 & 3,78 & 3,99 & 2,04 & 2,51 & 2,20 \\
\hline DP & 1,38 & 1,33 & 1,37 & 1,04 & 1,13 & 1,09 \\
\hline$a$ & 0,73 & 0,73 & 0,73 & 0,71 & 0,72 & 0,73 \\
\hline \multicolumn{4}{|c|}{ Médias } & \multicolumn{3}{|c|}{ Médias } \\
\hline Percentil & Mulher & Homem & Todos & Mulher & Homem & Todos \\
\hline 5 & 1,80 & 1,60 & 1,80 & 1,00 & 1,00 & 1,00 \\
\hline 10 & 2,20 & 2,00 & 2,20 & 1,00 & 1,20 & 1,00 \\
\hline 15 & 2,60 & 2,20 & 2,40 & 1,00 & 1,20 & 1,00 \\
\hline 20 & 2,80 & 2,60 & 2,80 & 1,00 & 1,40 & 1,20 \\
\hline 25 & 3,00 & 2,80 & 3,00 & 1,20 & 1,60 & 1,20 \\
\hline 30 & 3,40 & 3,00 & 3,20 & 1,20 & 1,80 & 1,40 \\
\hline 35 & 3,60 & 3,20 & 3,40 & 1,40 & 2,00 & 1,60 \\
\hline 40 & 3,80 & 3,40 & 3,60 & 1,60 & 2,00 & 1,60 \\
\hline 45 & 4,00 & 3,60 & 3,80 & 1,60 & 2,20 & 1,80 \\
\hline 50 & 4,20 & 3,80 & 4,00 & 1,80 & 2,40 & 2,00 \\
\hline 55 & 4,22 & 4,00 & 4,20 & 2,00 & 2,60 & 2,20 \\
\hline 60 & 4,60 & 4,20 & 4,40 & 2,00 & 2,60 & 2,20 \\
\hline 65 & 4,60 & 4,27 & 4,60 & 2,20 & 2,80 & 2,40 \\
\hline 70 & 5,00 & 4,40 & 4,80 & 2,40 & 3,00 & 2,60 \\
\hline 75 & 5,20 & 4,60 & 5,00 & 2,60 & 3,20 & 2,80 \\
\hline 80 & 5,40 & 5,00 & 5,20 & 3,00 & 3,40 & 3,20 \\
\hline 85 & 5,60 & 5,20 & 5,40 & 3,20 & 3,80 & 3,40 \\
\hline 90 & 6,00 & 5,60 & 5,80 & 3,60 & 4,00 & 3,80 \\
\hline 95 & 6,40 & 6,00 & 6,40 & 4,00 & 4,60 & 4,40 \\
\hline
\end{tabular}

similares aos encontrados para a versão estendida do instrumento, a ECR-Brasil, de Shiramizu et al. (2013). Dos 20 coeficientes comparados entre os dois instrumentos, dois se mostraram diferentes. A similaridade entre a maioria dos coeficientes de correlação testados para a medida reduzida e estendida confere ao instrumento reduzido relativa equivalência ao instrumento estendido de Shiramizu et al. (2013). A amostra dos dois estudos é diferente, principalmente no que diz respeito à idade, e isso pode contribuir com as explicações para as diferenças entre os dois instrumentos nos dois coeficientes de correlação. Ainda assim, salienta-se a importância do uso do instrumento estendido, sobretudo se o foco principal da pesquisa for o "apego", em função dos maiores índices de consistência interna da escala, se comparada à versão reduzida.
Também se verificou que a ECR-R-Brasil foi capaz de diferenciar grupos de pessoas que estavam em relacionamento amoroso compromissado daquelas que em relacionamento sem compromisso. Em acordo com a hipótese inicial e com os resultados encontrados para a medida estendida no Brasil (Shiramizu et al., 2013), as pessoas em relacionamento amoroso compromissado apresentaram menores níveis de ansiedade e evitação relacionadas ao apego. Esses resultados, somados às correlações com sociossexualidade, fortalecem a noção de que pessoas com características de apego seguro (baixos níveis de ansiedade e evitação) tendem a estabelecer relacionamentos amorosos mais duradouros e estáveis (Collins \& Read, 1990; Hazan \& Shaver, 1987). Constataram-se também diferenças sexuais nas dimensões de apego em acordo com estudos nacionais 
e internacionais (Del Giudice, 2009, 2011; Shiramizu et al., 2013).

Por fim, a tabela de normas para a amostra favorece a comparação de resultados de pesquisas futuras que usem essa escala. Considerando a amplitude da amostra deste estudo e o fato de haver participantes de todo o
Brasil, podem-se considerar essas normas como úteis para o contexto nacional. Contudo, ressalta-se a necessidade de observação da escolaridade dos participantes em possíveis estudos comparativos, tendo em vista que a amostra normativa foi quase pela metade composta por pessoas com ensino superior completo.

\section{An attachment measure: Brazilian version of Experiences in Close Relationship Scale - Short Form}

Abstract: The aim of this study was to adapt and seek validity evidence for the Brazilian population of the short version of the Experience in Close Relationship Scale. After translation procedures of the 12 items, 4,879 adults from all regions of the country answered the instrument, being $66 \%$ women. Exploratory and confirmatory factorial analyses showed a suitable two-factor structure, in line with the original study. The reliability coefficients of "anxiety" and "avoidance" factors were adequate. We found results of sex differences and correlations with length of relationship and sociosexuality according to the theoretical assumptions and very similar to those found in the Brazilian long-version of the measure. Also, the scale was able to discriminate participants who were in a committed relationship of those in non-committed relationships. We consider that our Brazilian adapted version of the instrument displayed satisfactory evidence of validity and reliability.

Keywords: attachment, attachment behavior, love, psychological testing, test validity.

\section{Une mesure de l'attachement: version brésilienne de la Experiences in Close Relationship Scale - Réduit (ECR-R-Brésil)}

Résumé: Les objectifs de cette étude étaient d'adapter et de trouver des preuves de validité pour la population brésilienne de la version réduite de l'échelle Experience in Close Relationship. Après la traduction de ses 12 questions, l'instrument a été répondu par 4,879 adultes de toutes les régions du pays, dont $66 \%$ femmes. Analyses factorielles exploratoires et confirmatoires ont montré la pertinence de la structure de deux facteurs, conformément l'étude originale. La fiabilité de les facteurs « anxiété » et « evitement » étaient appropriées. Les résultats pour les différences de sexe et corrélations avec la durée de la relation et socioséxualité ont respecté les hypothèses théoriques et ont été très semblables à celles trouvées par la version brésilienne étendue de la mesure. L'échelle a été capable de distinguer les participants qui étaient dans une relation engagée de ceux qui étaient dans relations sans engagement. On considère que cette version brésilienne adaptée de l'instrument a montré preuves satisfaisantes de validité et fiabilité.

Mots-clés: attachement, comportement d'attachement, amour, tests psychologiques, validation du test.

\section{Una medida del apego: versión brasileña de la Experiences in Close Relationship Scale - Reducida (ECR-R-Brasil)}

Resumen: Los objetivos de esta pesquisa fueron adaptar y buscar pruebas de validez para la población brasileña de la versión reducida de la escala Experience in Close Relationship. Después de procedimientos de traducción de los 12 ítems, el instrumento fue contestado por 4.879 adultos de todas las regiones del país, y $66 \%$ de ellos eran mujeres. Análisis factoriales exploratorias y confirmatorias demostraron la adecuación de la estructura de dos factores, en conformidad con el estudio original. Los índices de consistencia interna de los factores "ansiedad"y "evitación" se mostraron satisfactorios. Encontramos resultados de diferencias sexuales y correlaciones con el tiempo de relación y niveles de sociosexualidad de acuerdo con las hipótesis teóricas y muy similares a los encontrados para la versión brasileña de la medida extendida. La escala fue capaz de distinguir los participantes que se encontraban en relaciones comprometidas de aquellos en relaciones sin compromiso. Se consideran satisfactorias las pruebas de validez y confiabilidad encontradas para la ECR-R-Brasil.

Palabras clave: apego, conducta de apego, amor, pruebas psicológicas, validez de la prueba. 


\section{Referências}

Ainsworth, M. D. S. (1989). Attachments beyond infancy. American Psychologist, 44(4), 709-716. doi: 10.1037/0003066X.44.4.709.

Allen, E. S., \& Baucom, D. H. (2004). Adult attachment and patterns of extradyadic involvement. Family Process, 43(4), 467-488. doi: 10.1111/j.1545-5300.2004.00035.x.

Alonso-Arbiol, I., Balluerka, N., \& Shaver, P. (2007). A Spanish version of the Experiences in Close Relationships (ECR) adult attachment questionnaire. Personal Relationships, 14(1), 45-63. doi: 10.1111/j.1475-6811.2006.00141.x.

Bowlby, J. (1998). Apego e perda: vol. III. Perda: tristeza e depressão (2a ed., V. Dutra, trad.). São Paulo, SP: Martins Fontes. (Trabalho original publicado em 1980).

Bowlby, J. (2002). Apego e perda: vol I. Apego (3a ed., Á. Cabral, trad.). São Paulo, SP: Martins Fontes. (Trabalho original publicado em 1969).

Bowlby, J. (2004). Separação: angústia e raiva (4a ed., L. Regenberg, O. S. da Mota \& M. Hegenberg, trads.). São Paulo, SP: Martins Fontes. (Trabalho original publicado em 1973).

Brennan, K. A., Clark, C. L., \& Shaver, P. R. (1998). Selfreport measurement of adult attachment: An integrative overview. In J. A. Simpson, \& W. S. Rholes (Eds.), Attachment theory and close relationships (pp. 46-76). New York, NY: Guilford.

Byrne, B. M. (2009). Structural equation modeling with AMOS: Basic concepts, applications, and programming (2nd ed.). New York, NY: Routledge.

Collins, N. L., \& Read, S. J. (1990). Adult attachment, working models and relationship quality in dating couples. Journal of Personality and Social Psychology, 58(4), 644-663. doi: 10.1037/0022-3514.58.4.644.

Cronbach, L. (1951). Coefficient alpha and the internal structure of tests. Psychometrika, 16(3), 297-334. doi: 10.4135/9781412961288.

Crowne, D., \& Marlowe, D. (1960). A new scale of social desirability independent of psychopathology. Journal of Consulting Psychology, 24(4), 349-354. doi: 10.1037/ h0047358.

Del Giudice, M. (2009). Sex, attachment, and the development of reproductive strategies. Behavioral and Brain Sciences, 32(1), 1-21. doi: 10.1017/s0140525x09000016.

Del Giudice, M. (2011). Sex differences in romantic attachment: A meta-analysis. Personality and Social Psychology Bulletin, 37(2), 193-214. doi: 10.1177/0146167210392789.

Feeney, J. A. (2008). Adult romantic attachment: Developments in the study of couple relationships. In J. Cassidy \& P. R. Shaver (Eds.), Handbook of Attachment: Theory, Research, and Clinical Applications (pp. 456-481). New York, NY: Guilford.

Feeney, J. A., \& Noller, P. (1990). Attachment style as a predictor of adult romantic relationships. Journal of Personality and Social Psychology, 58(2), 281. doi: 10.1037//0022-3514.58.2.281.
Fisher, R. A. (1921). On the probable error of a coefficient of correlation deduced from a small sample. Metron, 1, 3-32.

Fraley, R. C., \& Shaver, P.R. (2000). Adultromantic attachment: Theoretical developments, emerging controversies, and unanswered questions. Review of General Psychology, 4, 132-154. doi: 10.1037//1089-2680.4.2.132.

Gentzler, A. L., \& Kerns, K. A. (2004). Associations between insecure attachment and sexual experiences. Personal Relationships, 11, 249-265. doi: 10.1111/j.14756811.2004.00081.x.

Hazan, C., \& Shaver, P. R. (1987). Romantic love conceptualized as an attachment process. Journal of Personality and Social Psychology, 52(3), 511-524. doi: 10.1037//0022-3514.52.3.511.

Jackson, D. L., Gillaspy Jr., J. A., \& Purc-Stephenson, R. (2009). Reporting practices in confirmatory factor analysis: An overview and some recommendations. Psychological Methods, 14(1), 6-23. doi: 10.1037/a0014694.

Jackson, J. J., \& Kirkpatrick, L. A. (2007). The structure and measurement of human mating strategies: Toward a multidimensional model of sociosexuality. Evolution and Human Behavior, 28(6), 382-391. doi: 10.1016/j. evolhumbehav.2007.04.005.

Kirkpatrick, L. A., \& Hazan, C. (1994). Attachment styles and close relationships: A four-year prospective study. Personal Relationships, 1(2), 123-142. doi: 10.1111/ j.1475-6811.1994.tb00058.x.

Mallinckrodt, B., \& Wang, C-C. (2004). Quantitative methods for verifying semantic equivalence of translated research instruments: A Chinese version of the Experiences in Close Relationships Scale. Journal of Counseling Psychology, 51(3), 368-379. doi: 10.1037/0022-0167.51.3.368.

Natividade, J. C., Fernandes, H. B. F., \& Hutz, C. S. (2013). Evidências de validade para o Brasil do Inventário de Orientação Sociossexual Revisado (SOI-R-Brasil). Pôster apresentado no VI Congresso Brasileiro de Avaliação Psicológica, Maceió, AL.

Nunnally, J. C. (1978). Psychometric Theory (2nd ed.). Nova York, NY: McGraw Hill.

Paiva, C. A., \& Figueiredo, B. (2010). Study of validation of the Portuguese version of the inventory Experiences in Close Relationships. Interpersona, 4(2), 237-270. doi: 10.5964/ijpr.v4i2.51.

Penke, L., \& Asendorpf, J. B. (2008). Beyond global sociosexual orientations: a more differentiated look at sociosexuality and its effects on courtship and romantic relationships. Journal of Personality and Social Psychology, 95(5), 1113. doi: 10.1037/t05284-000.

Picardi, A., Bitetti, D., Puddu, P., \& Pasquini, P. (2000). La scala "Experiences in close relationships" (ECL), un nuovo strumento per la valutazione dell'attaccamento negli adulti: traduzione, adattamento e validazione della versione italiana. Rivista di Psichiatria, 35(3), 114-120.

Ribas Jr, R. D. C., Moura, M. L. S., \& Hutz, C. S. (2004). Adaptação brasileira da escala de desejabilidade 
social de Marlowe-Crowne. Avaliação Psicológica, $3(2), 83-92$.

Schmitt, D. P. (2005). Sociosexuality from Argentina to Zimbabwe: A 48-nation study of sex, culture, and strategies of human mating. Behavioral and Brain Sciences, 28(2), 247-275. doi: 10.1017/s0140525x05000051.

Shaver, P. R., Hazan, C., \& Bradshaw, D. (1988). Love as attachment: The integration of three behavioral systems. In R. J. Sternberg, \& M. L. Barnes (Eds.), The psychology of love (pp. 68-99). New Haven, CT: Yale University Press.

Shiramizu, V. K. M., Natividade, J. C., \& Lopes, F. A. (2013). Evidências de validade do Experience in Close Relationships (ECR) Inventory para o Brasil. Estudos de Psicologia (Natal), 18(3), 457-465. doi: 10.1590/s1413$294 \times 2013000300006$.

Simpson, J. A. (1990). Influence of attachment styles on romantic relationships. Journal of Personality and
Social Psychology, 59(5), 971. doi: 10.1037//00223514.59.5.971.

Wachelke, J., Natividade, J. C., Andrade, A. D., Wolter, R., \& Camargo, B. (2014). Caracterização e avaliação de um procedimento de coleta de dados online (CORP). Avaliação Psicológica, 13(1), 143-146.

Wei, M., Russell, D. W., Mallinckrodt, B., \& Vogel, D. L. (2007). The Experiences in Close Relationship Scale (ECR)-short form: Reliability, validity, and factor structure. Journal of Personality Assessment, 88(2), 187204. doi: 10.1080/00223890701268041.

Recebido: 02/09/2014

Revisado: 03/01/2015

Aceito: 19/02/2015 


\section{Anexo 1}

Versão brasileira da Experience in Close Relationship - Reduzida (ECR-R-Brasil)

Por favor, leia as afirmações abaixo e marque o quanto cada uma descreve as emoções e sentimentos que você geralmente tem em relacionamentos amorosos e/ou sexuais. Queremos saber como você se sente em relacionamentos amorosos e/ou sexuais de modo geral, não apenas no seu relacionamento atual ou no seu último relacionamento. Mesmo que você nunca tenha tido um relacionamento, por favor, responda imaginando como você se sentiria se estivesse em um. Responda o quanto você concorda com as frases abaixo. Observe que quanto mais próximo de 1 você marcar, menos você concorda com a afirmação; quanto mais próximo de 7 você marcar, mais você concorda com a afirmação.

\begin{tabular}{|c|c|c|c|c|c|c|c|}
\hline & $\begin{array}{l}\text { Discordo } \\
\text { totalmente }\end{array}$ & & & Neutro & & & $\begin{array}{l}\text { Concordo } \\
\text { totalmente }\end{array}$ \\
\hline $\begin{array}{l}\text { 1. Ajuda muito poder contar com meu(minha) parceiro(a) } \\
\text { em momentos de necessidade. }\end{array}$ & 1 & 2 & 3 & 4 & 5 & 6 & 7 \\
\hline $\begin{array}{l}\text { 2. Eu preciso de muitas garantias de que sou amado por } \\
\text { meu(minha) parceiro(a). }\end{array}$ & 1 & 2 & 3 & 4 & 5 & 6 & 7 \\
\hline $\begin{array}{l}\text { 3. Eu recorro ao(à) meu(minha) parceiro(a) para muitas } \\
\text { coisas, incluindo para conforto e segurança emocional. }\end{array}$ & 1 & 2 & 3 & 4 & 5 & 6 & 7 \\
\hline $\begin{array}{l}\text { 4. Frequentemente, eu acho que meu(minha) parceiro(a) não } \\
\text { quer tanta proximidade afetiva quanto eu gostaria. }\end{array}$ & 1 & 2 & 3 & 4 & 5 & 6 & 7 \\
\hline $\begin{array}{l}\text { 5. Geralmente, tento evitar muita proximidade afetiva com } \\
\text { meu(minha) parceiro(a) }\end{array}$ & 1 & 2 & 3 & 4 & 5 & 6 & 7 \\
\hline $\begin{array}{l}\text { 6. Às vezes, meu desejo de ficar muito próximo } \\
\text { afetivamente acaba assustando as pessoas. }\end{array}$ & 1 & 2 & 3 & 4 & 5 & 6 & 7 \\
\hline $\begin{array}{l}\text { 7. Eu costumo conversar sobre os meus problemas e } \\
\text { preocupações com meu(minha) parceiro(a). }\end{array}$ & 1 & 2 & 3 & 4 & 5 & 6 & 7 \\
\hline $\begin{array}{l}\text { 8. Eu fico frustrado se meu(minha) parceiro(a) não está } \\
\text { disponível quando eu preciso dele(a). }\end{array}$ & 1 & 2 & 3 & 4 & 5 & 6 & 7 \\
\hline $\begin{array}{l}\text { 9. Eu fico preocupado quando meu(minha) parceiro(a) fica } \\
\text { muito próximo afetivamente de mim. }\end{array}$ & 1 & 2 & 3 & 4 & 5 & 6 & 7 \\
\hline $\begin{array}{l}\text { 10. Preocupa-me que meu(minha) parceiro(a) não se importe } \\
\text { comigo tanto quanto eu me importo com ele(a). }\end{array}$ & 1 & 2 & 3 & 4 & 5 & 6 & 7 \\
\hline
\end{tabular}

\section{Cálculo das dimensões}

Ansiedade: calcular média aritmética dos itens pares (2, 4, 6, 8 e 10);

Evitação: inverter itens 1,3 e 7 e calcular média aritmética dos itens ímpares. 